\title{
Optimization Design of Stainless Steel Stamping Multistage Pump Based on Orthogonal Test
}

\author{
Shi Weidong, Wang Chuan, Lu Weigang, Zhou Ling and Zhang Li \\ Research Center of Fluid Machinery Engineering and Technology, Jiangsu University, \\ Xuefu Road, Zhenjiang-city, 086-212013, China, wangchuan198710@126.com
}

\begin{abstract}
Stainless steel stamping multistage pump has become the mainstream of civil multi-stage pump. Combined with the technological features of stamping and welding pump, the studies of design for hydraulic parts of pump were come out. An $\mathrm{L}_{18}\left(3^{7}\right)$ orthogonal experiment was designed with seven factors and three values including blade inlet angle, impeller outer diameter, guide vane blade number, etc. 18 plans were designed. The two stage of whole flow field on stainless steel stamping multistage pump at design point for design was simulated by CFD. According to the test result and optimization design with experimental research, the trends of main parameters which affect hydraulic performance were got. After being manufactured and tested, the efficiency of the optimal model pump reaches $61.36 \%$ and the single head is more than $4.8 \mathrm{~m}$. Compared with the standard efficiency of $53 \%$, the design of the stainless steel stamping pump is successful. The result would be instructive to the design of Stainless steel stamping multistage pump designed by the impeller head maximum approach.
\end{abstract}

Key words: stamping and welding pump; orthogonal experiments; internal flows; numerical simulation.

\section{Introduction}

Most pumps in the past were manufactured by casting process while existing pump designs are based on the development of casting pumps. The structure and process of the prevailing stainless steel stamping pumps are very different from casting pumps. There are two main structural parameters which will affect the pump performance .Firstly, the vanes of stamping pumps are much thinner than cast pumps'; secondly, the width of axial runners of stamping pumps is much smaller than the casting pumps'[1]. This study attempted to study the designs of stainless steel stamping pumps by using the structural characteristics.

The design of stamping pump is a combination of hydraulic design and processing technology design. On the premise of production process, the design parameters of the key hydraulic components of stamping pump are analyzed through the CFD techniques and orthogonal experiments, we get the trends of main parameters which affect hydraulic performance and finally design the stamping multistage pump which is efficient and has a widely effective district.

\section{Orthogonal test design of stainless steel stamping multistage pumps.}

Orthogonal test design is a kind of scientific method of arranging and analyzing multiple factors, by using a orthogonal table which is made according to the principle of "the orthogonal" of mathematics, it will make scientific arrangement of the test plan and analyze the test result. In conclusion, the mathematical method of optimal production conditions and process conditions will be found out[2].

\subsection{Test purposes.}

Explore the law how various geometric parameters of stainless steel stamping pumps affect the efficiency and head of rated point.

\subsection{Test index.}

Mainly inspects the efficiency $\eta$ at the rated flow of $Q=8 \mathrm{~m}^{3} / \mathrm{h}, n=2850 \mathrm{r} / \mathrm{min}, H=45 \mathrm{~m}$, stage number $i=10$ and puts forward the optimal design efficiency.

Accepted for publication December 4 2010: Paper number O10036S

Corresponding author: Shi Weidong, Professor,wdshi@ujs.edu.cn.

This paper was presented at 2010 International Conference on Pumps and Fans, October 18-21, 2010, Hangzhou, China 


\subsection{Determine the test factors.}

The efficiency of pumps is jointly determined by impellers and guide vanes, so the orthogonal experiment integrates the structure parameters of impellers and guide vanes and selects the following seven groups of geometric parameters as test factors: $D_{2 m i n}$ (outer diameter of impeller rear floor), $\beta_{1}$ (blade inlet angle, $\beta_{2}$ (blade outlet angle, $b_{2}$ (blade outlet width), $B_{3}$ (guide vane inlet width), $Z_{1}$ (impeller blade number), $Z_{2}$ (guide vane blade number). It is showed in figure 1 and 2.

Firstly devise the impeller of stamping multistage pump by adopting the impeller maximal diameter design method, in order to get largest single head, the outer diameter of front floor of impeller is $\varphi 79.5 \mathrm{~mm}$ which is a litter shorter than the diameter of inner wall and the outlet of impeller is skewed.

The reasons why I don't choose other geometric parameters as the experimental factors are as follows:

(1) Impeller inlet diameter $D_{j}$ When impeller inlet diameter increases, the impeller inlet velocity can decrease, thereby improving the pump's anti-cavitations' performance. But the area of sealing ring can also increase, which can lower the pump volume efficiency. The pump is mainly used in deep wells, regardless of the anti-cavitations performance. In order to improve pump efficiency, a smaller value can be taken after the initial calculation of impeller inlet diameter.

(2) Blade inlet diameter $D_{1}$ Reducing the blade inlet diameter will accordingly increase the flow length. On the premise of meeting the manufacturability, protract and thin the blade inlet, which will not only improve the stability of the lift curve, but also increase the pump efficiency slightly.

(3) Guide vane inlet angle $\beta_{3}$ Guide vane inlet angle and guide vane inlet width determine the flow area of guide vane import together. By adjusting the $B_{3}$, you can indirectly adjust the $\beta_{3}$ which affects the pump performance. And adjusting guide vane inlet width is more quickly and can save lots of work. So do not choose the guide vane inlet angle $\beta_{3}$ as experimental factor.
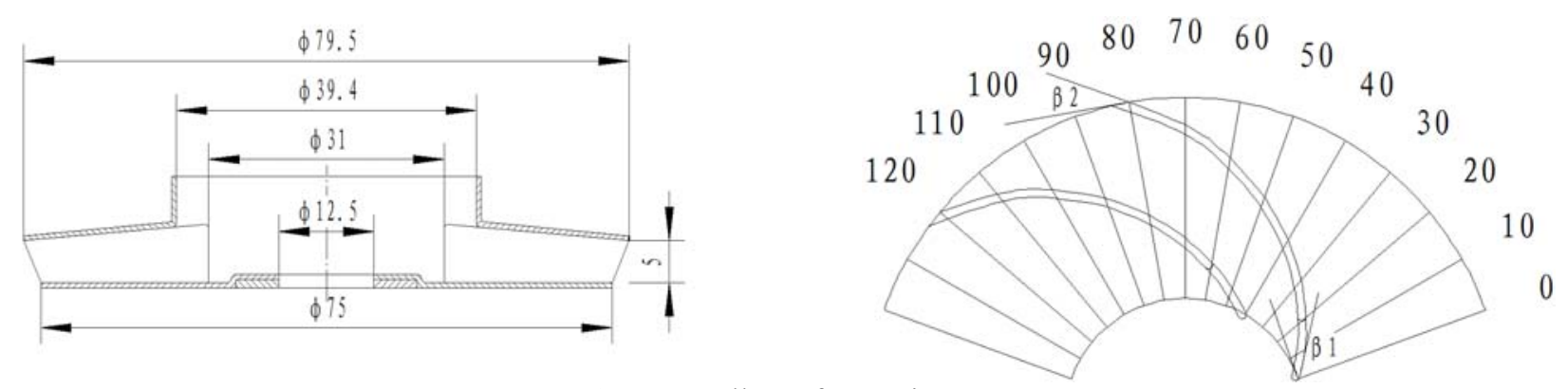

Fig. 1 Impellers of stamping pumps
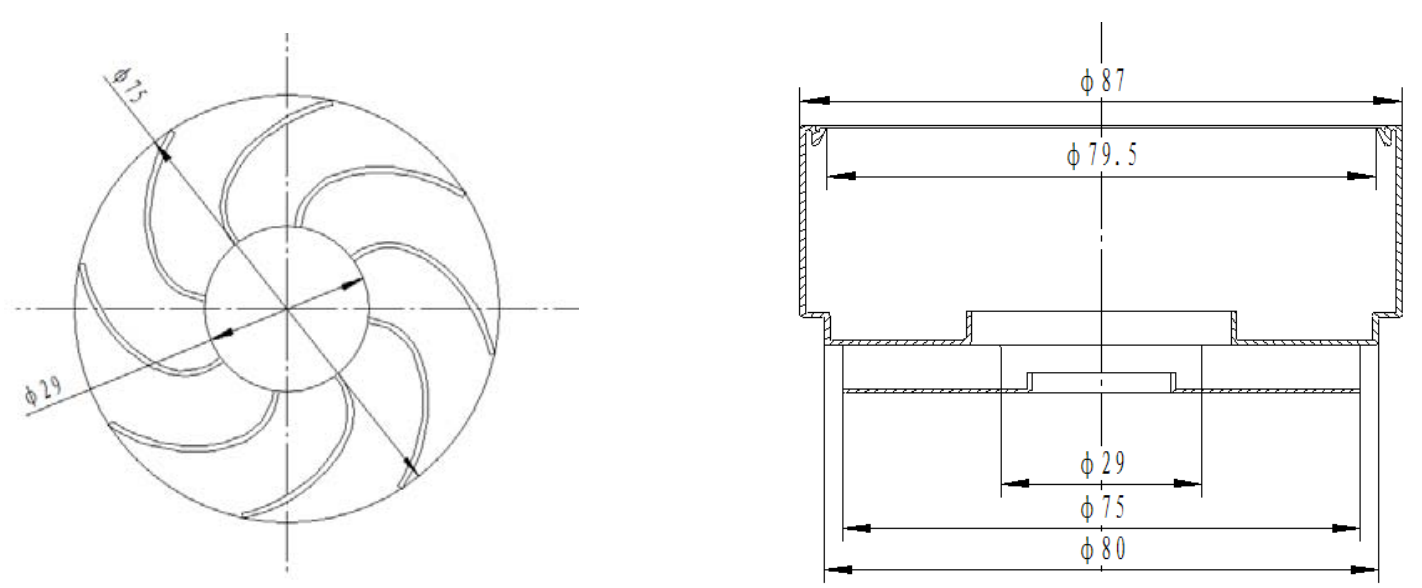

Fig. 2 Draft tubes of stamping pumps

\subsection{Factors level.}

The design parameters of model pump are as follows: $Q=8 \mathrm{~m}^{3} / \mathrm{h}, H=45 \mathrm{~m}, i=10, n=2850 \mathrm{r} / \mathrm{min}, \eta=53 \%$. According to the specific speed $n_{s}=158$, relevant geometric parameters are determined and three levels are chosen, which is showed in table 1 and table 2 .

Table 1 Orthogonal experimental factors

\begin{tabular}{|c|c|c|c|c|c|c|c|}
\hline \multicolumn{8}{|c|}{ Factors } \\
\hline 1 & 75 & 25 & 4 & 5.2 & 35 & 7 & 7 \\
\hline 3 & 79.5 & 40 & 6 & 7.2 & 65 & 11 & 9 \\
\hline
\end{tabular}


Table 2 Test scheme

\begin{tabular}{|c|c|c|c|c|c|c|c|}
\hline No. & $A$ & $B$ & $C$ & $D$ & $E$ & $F$ & $G$ \\
\hline 1 & 75 & 34 & 4 & 6.2 & 65 & 7 & 7 \\
\hline 2 & 75 & 25 & 6 & 5.2 & 50 & 9 & 8 \\
\hline 3 & 75 & 40 & 5 & 7.2 & 35 & 11 & 9 \\
\hline 4 & 77 & 25 & 5 & 6.2 & 50 & 9 & 9 \\
\hline 5 & 77 & 34 & 6 & 7.2 & 65 & 7 & 8 \\
\hline 6 & 77 & 40 & 4 & 5.2 & 35 & 11 & 7 \\
\hline 7 & 79.5 & 34 & 6 & 6.2 & 50 & 7 & 7 \\
\hline 8 & 79.5 & 40 & 5 & 5.2 & 35 & 9 & 8 \\
\hline 9 & 79.5 & 25 & 4 & 7.2 & 65 & 11 & 9 \\
\hline 10 & 75 & 25 & 6 & 7.2 & 35 & 11 & 8 \\
\hline 11 & 75 & 40 & 4 & 5.2 & 65 & 7 & 9 \\
\hline 12 & 75 & 34 & 5 & 6.2 & 50 & 9 & 7 \\
\hline 13 & 77 & 34 & 4 & 7.2 & 35 & 7 & 8 \\
\hline 14 & 77 & 25 & 5 & 6.2 & 65 & 9 & 7 \\
\hline 15 & 77 & 40 & 6 & 5.2 & 50 & 11 & 9 \\
\hline 16 & 79.5 & 25 & 6 & 6.2 & 65 & 7 & 9 \\
\hline 17 & 79.5 & 34 & 4 & 7.2 & 50 & 11 & 8 \\
\hline 18 & 79.5 & 40 & 5 & 5.2 & 35 & 9 & 7 \\
\hline
\end{tabular}

\section{The intuitive analysis of orthogonal test.}

Considering 18 groups of impellers and guide vanes which are made from prototype manufacturing will not only need an amount of money of mould processing and long test cycle, but also biggish manufacturing errors and test errors inevitably exist in large amount of prototype tests. In combination with consideration that current computational fluid dynamics (CFD) technology has been able to reflect truly the internal flow field distribution of centrifugal pumps and predict accurately the head, efficiency and shaft power of pumps at rated conditions [3]. Therefore, by using flow analysis results, forecast preliminarily whether the pump performances meet the design requirements.

\subsection{Create 3d model.}

The simulation flowing parts of stainless steel stamping multistage pumps are composed by inlet section, two impellers, two guide vanes and outlet section. The solid models are respectively established and assembled together in Pro-E. The $3 \mathrm{~d}$ model of the whole flow field is showed in figure 3 . The quality of $3 \mathrm{~d}$ model will directly affect the division of grid, so avoid producing small and discontinuous surfaces when creating $3 \mathrm{~d}$ model. In the numerical simulations on stamping multistage pumps, this research introduces two stages whole flow field of real significance. The model includes the liquid of two impellers, two guide vanes and cavity of the front and rear cover, the whole flow passage is not almost simplified. The import and export sections is enough outspread, which makes the inlet and outlet boundary not affected by the import and export flow.

\subsection{Model mesh.}

Put the $3 \mathrm{~d}$ model into Gambit and generate the whole flow passage by using Booleans. Due to the great pressure gradient in the blades of impeller, firstly make division of blade surfaces with smaller size of face mesh, then divide the two impellers and two guide vanes using hybrid grid, lastly divide the import and export sections with structured grid. It is showed in figure 4.

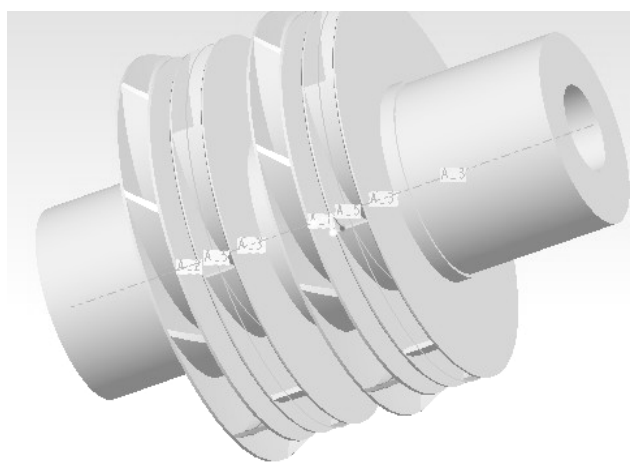

Fig. 3 3D entity mode

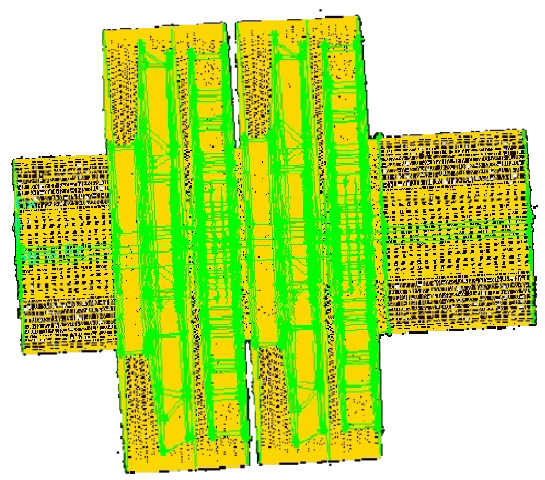

Fig. 4 Grid mode

\subsection{Control equations and boundary conditions}

When making simulation calculations by CFD, use standard $\mathrm{k}-\varepsilon$ turbulence model control equations and SIMPLEC algorithm, besides, momentum, turbulent kinetic energy and turbulence dissipation rate all adopt second order upwind, moreover, pressure item selects standard. In order to accelerate convergence, various relaxation factors employ 0.3 . The inlet and outlet of pump respectively adopt velocity inlet and outflow, the solid wall is not slip, namely relative velocity $\mathrm{w}=0$ [4]. 


\subsection{Results and analysis.}

Through the numerical simulation of whole flow field on the scheme that 18 groups of impellers and guide vanes coordinate with each other, The single head $H$ and efficiency $\eta$ are get and listed in table 3 .

Table 3 Summary of test results

\begin{tabular}{c|l|l|l|l|l|l|l|l|l}
\hline No & 1 & 2 & 3 & 4 & 5 & 6 & 7 & 8 & 9 \\
\hline$H / m$ & 3.67 & 4.53 & 5.30 & 4.48 & 4.56 & 4.45 & 4.71 & 5.07 & 3.71 \\
\hline$\eta / \%$ & 53.8 & 58.9 & 58.2 & 59.7 & 52.7 & 54.3 & 52.0 & 55.5 & 50.5 \\
\hline No & 10 & 11 & 12 & 13 & 14 & 15 & 16 & 17 & 18 \\
\hline$H / m$ & 5.21 & 3.76 & 4.53 & 4.22 & 4.45 & 5.57 & 4.61 & 4.41 & 4.96 \\
\hline$\eta / \%$ & 59.4 & 51.8 & 57.8 & 59.1 & 55.0 & 54.0 & 51.8 & 51.1 & 54.9 \\
\hline
\end{tabular}

The calculation and analysis of orthogonal experimental result are as follows:

The head index at rated point

Column 1:

$K_{1 A}=3.67+4.53+5.30+5.21+3.76+4.53=27$

$K_{2 A}=4.48+4.56+4.45+4.22+4.45+5.57=27.73$

$K_{3 A}=4.71+5.07+3.71+4.61+4.41+4.96=27.47$

In the formula, $K_{1 A}, K_{2 A}, K_{3 A}$ respectively show the sum of simulation result when factor $A$ is 1,2 , and 3. To make comparison with A of different levels, $k$ is introduced.

$k_{1 A}=27 / 6=4.50$

$k_{2 A}=27.73 / 6=4.62$

$k_{3 A}=27.47 / 6=4.58$

In the formula, $k_{1 A}, k_{2 A}, k_{3 A}$ respectively show the single head at rated point when factor $A$ is 1,2 , and 3 .

The calculation methods of the remaining six columns are the same as the first column, and the results are filled in table 4 and 5.

$R$ in each column can be obtained by the maximum of $k_{1 A}, k_{2 A}$ and $k_{3 A}$ minus the minimum of $k_{1 A}, k_{2 A}$ and $k_{3 A}$.

For example:

Column 1: $R_{A}=\max \left\{k_{1 A}, k_{2 A}, k_{3 A}\right\}-\min \left\{k_{1 A}, k_{2 A}, k_{3 A}\right\}=4.62-4.50=0.12$

Table 4 Head results analysis at the rated point

\begin{tabular}{l|l|l|l|l|l|l|l}
\hline$H / m$ & $A$ & $B$ & $C$ & $D$ & $E$ & $F$ & $G$ \\
\hline$K_{I}$ & 27.0 & 27.0 & 24.21 & 28.33 & 29.20 & 25.53 & 26.21 \\
\hline$K_{2}$ & 27.73 & 26.09 & 28.80 & 26.45 & 28.22 & 28.02 & 28.54 \\
\hline$K_{3}$ & 27.47 & 29.11 & 29.18 & 27.40 & 24.77 & 28.64 & 27.43 \\
\hline$k_{l}$ & 4.50 & 4.50 & 4.04 & 4.72 & 4.87 & 4.25 & 4.37 \\
\hline$k_{2}$ & 4.62 & 4.35 & 4.80 & 4.41 & 4.70 & 4.67 & 4.76 \\
\hline$k_{3}$ & 4.58 & 4.85 & 4.86 & 4.57 & 4.13 & 4.77 & 4.57 \\
\hline$R$ & 0.12 & 0.5 & 0.82 & 0.31 & 0.74 & 0.52 & 0.39 \\
\hline
\end{tabular}

Table 5 Efficiency results analysis at the rated point

\begin{tabular}{l|l|l|l|l|l|l|l}
\hline$\eta / \%$ & $A$ & $B$ & $C$ & $D$ & $E$ & $F$ & $G$ \\
\hline$K_{1}$ & 339.9 & 335.3 & 320.6 & 329.4 & 341.4 & 321.2 & 324 \\
\hline$K_{2}$ & 334.8 & 326.5 & 341.1 & 330.1 & 333.5 & 341.8 & 340.5 \\
\hline$K_{3}$ & 315.8 & 328.7 & 328.8 & 331.0 & 315.6 & 327.5 & 326 \\
\hline$k_{1}$ & 56.65 & 55.88 & 53.43 & 54.9 & 56.9 & 53.53 & 54 \\
\hline$k_{3}$ & 55.8 & 54.42 & 56.85 & 55.02 & 55.58 & 56.97 & 56.75 \\
\hline$R$ & 52.63 & 54.78 & 54.8 & 55.17 & 52.6 & 54.58 & 54.33 \\
\hline
\end{tabular}

Generally speaking, the $\mathrm{R}$ of each column is different, because the size of $\mathrm{R}$ reflects the effect of various factors in tests. The bigger $\mathrm{R}$ is, the more relevant factor affects the index. As a result, the factor is usually regarded as a key factor. According to the size of $\mathrm{R}$ in table 4 and table 5 , the influence order of various factors can be obtained, which is showed in table 6 .

Table 6 Influence order of geometric parameters to performance

\begin{tabular}{l|l|l|l|l|l|l|l}
\hline Index & \multicolumn{9}{|c}{ High $\rightarrow$ Low } & $Z_{2}$ & $Z_{2}$ & $B_{3}$ & $D_{2 \min }$ \\
\hline$H$ & $b_{2}$ & $\beta_{1}$ & $Z_{1}$ & $\beta_{2}$ & $Z_{2}$ & $B_{3}$ \\
\hline$\eta$ & $\beta_{1}$ & $D_{2 \min }$ & $Z_{1}$ & $b_{2}$ & $Z_{2}$ & $\beta_{2}$ \\
\hline
\end{tabular}

Make comprehensive consideration and select the best scheme. 
The factor $A$ has the influence order on head as $A_{1} A_{2} A_{3}$ and efficiency as $A_{1} A_{2} A_{3}$; the factor $B$ has the influence order on head as $B_{3} B_{1} B_{2}$ and efficiency as $B_{1} B_{3} B_{2}$; the factor $C$ has the influence order on head as $C_{3} C_{2} C_{l}$ and efficiency as $C_{2} C_{3} C_{l}$; the factor $D$ has the influence order on head as $D_{1} D_{3} D_{2}$ and efficiency as $D_{3} D_{2} D_{1}$; the factor $E$ has the influence order on head as $E_{1} E_{2} E_{3}$ and efficiency as $E_{1} E_{2} E_{3}$; the factor $F$ has the influence order on head as $F_{3} F_{2} F_{1}$ and efficiency as $F_{2} F_{3} F_{1}$; the factor $G$ has the influence order on head as $G_{2} G_{3} G_{l}$ and efficiency as $G_{2} G_{3} G_{l}$.

To sum up, the best combination of head is $C_{3} E_{1} F_{3} B_{3} G_{2} D_{1} A_{2}$; the best combination of efficiency is $E_{1} A_{1} F_{2} C_{2} G_{2} B_{1} D_{3}$.

The purpose of this orthogonal experiment is that, under the premise of satisfying single head, the maximum efficiency at rated point can be obtained. So use the best combination of efficiency namely $E_{1} A_{1} F_{2} C_{2} G_{2} B_{1} D_{3}$. Through CFD predict the performance of the pump and obtain that the single head and efficiency respectively are $4.80 \mathrm{~m}$ and $63.2 \%$, which the head and efficiency both meet the design demands.

\section{Model test analysis.}

In order to verify whether the optimal stamping pump get the success, make an entity model and test the performance of the pump in the pump test bed of Jiangsu University. In the test the model pump runs well and the test data is showed in table 7. At the same time, make numerical simulation on the optimal stamping multistage pump at five points $(Q=3.2 、 Q=4.8, Q=6.4, Q=8$, $Q=9.6, Q=11.2 \mathrm{~m}^{3} / \mathrm{h}$ ), which is regarded as the basis of performance prediction. The result is showed in table 8 . The prediction value and test value of efficiency of the optimal pump can be seen in figure 5; the contrast of head (10 stages) is showed in figure $6[5]$.

Table 7 Impeller performance parameters $\quad(\mathrm{i}=10)$

\begin{tabular}{l|l|l|l|l}
\hline No. & $Q /\left(\mathrm{m}^{3} / \mathrm{h}\right)$ & $H /(\mathrm{m})$ & $P /(w)$ & $\eta /(\%)$ \\
\hline 1 & 0 & 60.25 & 1289.21 & 0 \\
\hline 2 & 3.12 & 57.45 & 1847.18 & 40.29 \\
\hline 3 & 4.02 & 56.59 & 2013.78 & 45.91 \\
\hline 4 & 5.15 & 55.70 & 2205.86 & 51.79 \\
\hline 5 & 6.11 & 54.44 & 2337.18 & 56.13 \\
\hline 6 & 7.11 & 52.16 & 2439.22 & 58.87 \\
\hline 8 & 8.16 & 48.42 & 2444.91 & 61.36 \\
\hline 9 & 9.20 & 41.79 & 2461.87 & 59.37 \\
\hline 10 & 10.33 & 32.68 & 2415.78 & 53.46 \\
\hline 11 & 11.19 & 24.17 & 2402.79 & 43.44 \\
\hline 12 & 12.19 & 13.96 & 2397.47 & 27.34 \\
\hline & 13.23 & 4.16 & 2428.44 & 8.72 \\
\hline
\end{tabular}

Table 8 Impeller performance parameters

\begin{tabular}{c|c|c|c}
\hline$Q /\left(\mathrm{m}^{3} / \mathrm{h}\right)$ & $H /(\mathrm{m})$ & $P /(\mathrm{W})$ & $\eta /(\%)$ \\
\hline 3.2 & 55.1 & 1010 & 48.6 \\
\hline 4.8 & 52.6 & 1280 & 54.7 \\
\hline 6.4 & 50.65 & 1510 & 59.5 \\
\hline 8 & 48 & 1690 & 63.2 \\
\hline 9.6 & 39.35 & 1760 & 59.6 \\
\hline 11.2 & 27 & 1759 & 47.6 \\
\hline
\end{tabular}

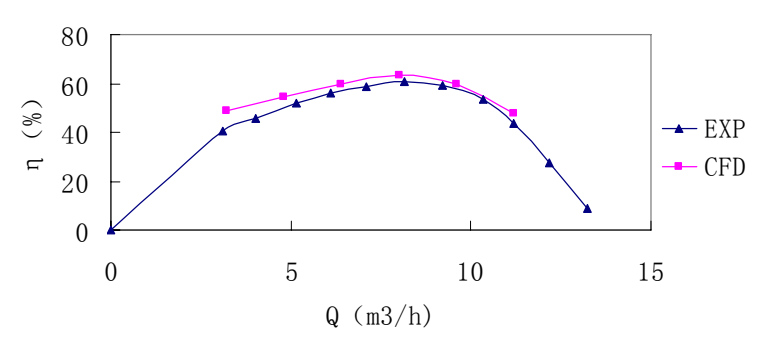

Fig. 5 Efficiency comparison of optimal program

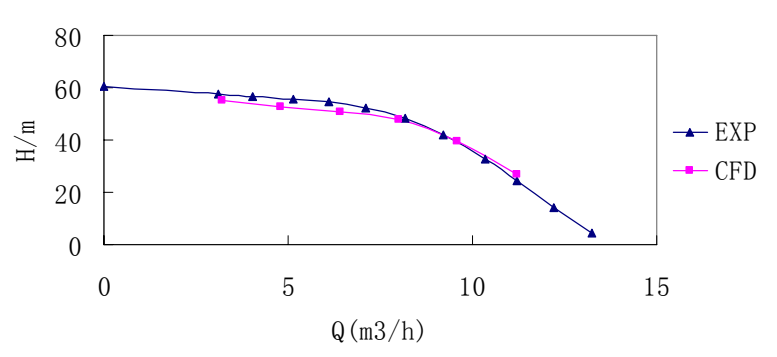

Fig. 6 Head comparison of optimal program

Compared with the performance curve of simulation value and experimental value, we can see that simulation value and experimental value coincide well in high efficiency zone, while in non-design condition, due to the back-flow in the impeller and guide vane, simulation value deflects experimental value. Therefore, the calculating method of numerical simulation on stainless steel stamping multistage pump at rated point is accurate [6]. From the figure 5 and 6 , the head curve of pump changes smoothly and the high efficiency zone is broad. By looking up the well pump standard of the People's Republic of China (GB/T 28162002), the efficiency of the pump 100QJ8 should be 53\%. Compared with the standard efficiency, the efficiency of the optimal model pump reaches $61.36 \%$ and the single head is more than $4.8 \mathrm{~m}$. At the same time, the efficiency of the same pump produced 
by Grundfos is $60 \%$, and the single head is only $4.2 \mathrm{~m}$. So the design of the stainless steel stamping pump is successful, then the optimal design of orthogonal test based on the CFD numerical simulation is successful too.

\section{Conclusion.}

(1) Using Fluent soft, make numerical simulations of two stages of whole flow field on 18 groups of experimental plans. By analyzing the efficiency and head and making comprehensive considerations, obtain that the influence order of geometric parameters to performance is $\beta_{1}, D_{2 \min }, Z_{1}, b_{2}, Z_{2}, \beta_{2}, B_{3}$.

(2) The purpose of this orthogonal experiment is that, under the premise of satisfying single head, the maximum efficiency at rated point can be obtained. So use the best combination of efficiency, namely blade inlet angle $\beta_{I}=35^{\circ}$; outer diameter of impeller rear floor $D_{2 \min }=75 \mathrm{~mm}$; impeller blade number $Z_{1}=9$; impeller blade outlet width $b_{2}=5 \mathrm{~mm}$; guide vane blade number $Z_{2}=8$; blade outlet angle $\beta_{2}=25^{\circ}$; guide vane inlet width $B_{3}=7.2 \mathrm{~mm}$.

(3) Through CFD make numerical simulations on the optimal plan of geometric parameters combination. Compared with the experimental result of model pump, we can see that simulation value and experimental value coincide well in high efficiency zone, which provides useful reference on the improvement of stainless steel stamping multistage pump.

\section{Acknowledgments}

The authors will show our special thanks to the supports by the research center of fluid machinery and technology from Jiangsu University.

\section{Nomenclature}

$\begin{array}{ll}Q & \text { Flow rate }\left[\mathrm{m}^{3}\right] \\ H & \text { Head }[\mathrm{m}] \\ n & \text { Rotation speed }[\mathrm{r} / \mathrm{min}] \\ P & \text { Power [w] } \\ \eta & \text { Efficiency [\%] } \\ M & \text { Moment [N.m] }\end{array}$

$\begin{array}{ll}b_{2} & \text { Blade outlet width [mm] } \\ B_{3} & \text { Guide vane inlet width }[\mathrm{mm}] \\ \beta_{1} & \text { blade inlet angle }\left[{ }^{\circ}\right] \\ \beta_{2} & \text { blade outlet angle }\left[{ }^{\circ}\right] \\ D_{2 \min } & \text { outer diameter of impeller rear floor[mm] } \\ Z & \text { blade number }\end{array}$

\section{References}

[1] Yao Chunling, Wang Chunhua, Liu Kun et al, 2006, "Characters and Current Developments of Stamping and Welding Centrifugal Pump," Manufacturing Automation (in Chinese), Vol. 28, pp. 214-215.

[2] Cong Xiaoqing, Yuan Shouqi, Yuan Danqing, 2005, "Research of Latin Square Test on Non-overload Sewage Pumps," Journal of Agricultural Machinery (in Chinese), Vol. 36, No. 10, pp. 66-69.

[3] Liu Yuanyi, Wang Guangye, 2007, “Computer-Aided Analysis on Inner flow in Stamping and Welding Multistage Centrifugal Pump's Impellers,” Chinese Journal of Mechanical Engineering(in Chinese), Vol. 43, No. 8, pp. $207-211$.

[4] Liu Yuanyi, Wang Guangye, 2006, “ Numerical Simulation of 3D Flow in Low specific Speed Stamping Multistage Pump's Impellers," Journal of Agricultural Machinery (in Chinese), Vol. 37, No. 11, pp. 60-62.

[5] Huang Si Sang Dike, Peng Shaohua et al, 2009, "Numerical Simulation and Experimental Verification of Hydraulic Performance of Stamping Multi-Pump,” Water-saving Irrigation (in Chinese), Vol. 12, pp. 48-51.

[6] Shi Weidong, Zhang Qihua, Lu Weigang, 2008, "Research on the Internal Flow of Impeller on New-type Deep Well Pump," Fluid Machinery(in Chinese), Vol. 36, No. 5, pp. 21-22. 